\title{
Uitsiton: wearable for mobility of the visually impaired
}

\section{Uitsiton: wearable para la movilidad de personas con discapacidad visual}

\author{
BUENDÍA-MARTÍNEZ, Adrian† \& GALLARDO-LÓPEZ, Lizbeth* \\ Universidad Autónoma Metropolitana. Avenida San Pablo 180, Azcapotzalco, Reynosa Tamaulipas, 02200 Mexico City, \\ Mexico.
}

ID $1^{\text {st }}$ Author: Adrian, Buendía-Martínez / ORC ID: 0000-0003-2031-3955

ID $1^{\text {st }}$ Coauthor: Lizbeth, Gallardo-López / ORC ID: 0000-0002-9254-8115, CVU CONACYT ID: 201883

DOI: $10.35429 /$ JSTA.2020.18.6.7.18

Received July 11, 2020; Acceptance December 30, 2020

\begin{abstract}
This article explains the development of the Uitsiton system, capable of detecting obstacles to alert visually impaired users, increasing their mobility and confidence when moving. Uitsiton is made up of a portable electronic device (wearable) and a mobile application (App) that operates under the Android platform. The wearable is integrated into a vest-like garment. It is used to compute the approximate distance between the visually impaired user and the obstacles that they can find in their path. The wearable covers a range of 180 degrees vertically and horizontally with respect to the center of the torso, and reaches a maximum distance of $120 \mathrm{~cm}$. The App works synchronously with the wearable, it receives a series of data corresponding to the measurements of the distance between the user and the obstacle, and it triggers a sound pattern and a vibration pattern according to the proximity of the obstacle.
\end{abstract}

Wearable, App, Mobility, Visually impaired

\section{Resumen}

Este artículo explica el desarrollo del sistema Uitsiton, capaz de detectar obstáculos para alertar a los usuarios con discapacidad visual, aumentando su movilidad y confianza al moverse. Uitsiton está compuesto por un dispositivo electrónico portátil (wearable) y una aplicación móvil (App) que funciona bajo la plataforma Android. El wearable está integrado en una prenda tipo chaleco y se utiliza para calcular la distancia aproximada entre el usuario con discapacidad visual y los obstáculos que pueden encontrar en su camino. El wearable cubre un rango de 180 grados vertical y horizontalmente con respecto al centro del torso, y alcanza una distancia máxima de $120 \mathrm{~cm}$. La App funciona de manera síncrona con el wearable; recibe una serie de datos correspondientes a las mediciones de la distancia entre el usuario y el obstáculo, y desencadena un patrón de sonido y un patrón de vibración acorde a la proximidad del obstáculo.

Wearable, App, Movilidad, Discapacidad visual

Citation: BUENDÍA-MARTÍNEZ, Adrian \& GALLARDO-LÓPEZ, Lizbeth. Uitsiton: wearable for mobility of the visually impaired. Journal of Scientific and Technical Applications. 2020. 6-18:7-18.

\footnotetext{
* Correspondence to Author (Email: glizbeth@azc.uam.mx)

$\dagger$ Researcher contributing as first author.
} 


\section{Introduction}

The World Health Organization (WHO, 2014) 10 facts about blindness and visual impairment report revealed that approximately 285 million people had a visual impairment in the year 2010; of which 39 million suffer from total blindness and 246 million have low vision. Visual impairment is a social problem because it makes autonomous mobility difficult and therefore their participation in meetings; for example, educational, work, cultural, among others. "Visual impairment can limit people's ability to perform everyday tasks and can affect their quality of life and ability to interact with the surrounding world." (WHO, 2014). Among the solutions to ensure that visually impaired people are able to move by themselves are mechanical prostheses; for example, the white cane and its multiple variations; in addition, automated prosthetics, such as smart clothing; specialized glasses, like proposed by Gonzales-Lorence et al., (2020); computer vision techniques, as proposed by Brito, G. J., (2020); among others; all of these solutions pursuit to make the visually impaired person feel emotionally secure and independent.

On one hand, according to Dakopoulos, D., \& Bourbakis, N. G. (2009), wearable computing is a branch of ubiquitous computing, where systems or devices can be worn with a specified purpose. The authors describe three categories for the obstacle detection: ETA (Electronic Travel Aids), EOA (Electronic Orientation Aids) and PLO (Position Locator Devices). The Electronic Travel Aids classification has also three system categories: Vision replacement, Vision enhancement and Vision substitution. On the other hand, according to Discant, A., et. al. (2007), the problem of obstacles detection has been treated in 4 approaches: SONAR, that is based on sound waves; RADAR, that is based on electromagnetic waves; LIDAR, that is based on light beam; and LADAR, that is based on laser beam. Thus, our system belongs to the ETA category and Vision substitution subcategory and employs the SONAR approach.

We build an experimental system, named Uitsiton, consisting of a portable electronic device (wearable) and a mobile App. Uitsiton's objective is to help visually impaired people to make their movements autonomously.
Wefocus on four aspects concerning usability: i) easy to learn, measured through learning time, ii) efficiency, measured through operating time, iii) sensing coverage range, and iv) scalability, measured by the possibility of incrementing the number of sensors. This experimental system is oriented to the internet of things (IoT).

We found four systems related to Uitsiton. The first one is the Wearable System Cardin, S., Thalmann, D., \& Vexo, F., (2007), which detects shoulder-height obstacles through ultrasonic range sensors. Wearable System notifies the user about the proximity of an object through the vibration of a vest; vibration takes place in a certain place(s) of the user's body. Vibration can be calibrated by synchronizing the vest with the PDA (Personal Digital Assistant); calibration depends on the sensitivity of each user. The operating time is not reported, but based on architecture, current consumption is estimated in a time of 10 hours. The data processing units are: a PIC16F87 microcontroller, a Bluetooth module (unspecified model) and a PDA. Learning time and scalability are not reported. This system is not oriented to the IoT.

The second one is the device Cinturón y Manillas Grijalva, H. M. \& Mejía, H. M., (2016), which detects obstacles at half-height in three directions (left, right and front), through ultrasonic range sensors. Cinturón y Manillas notifies the user of the proximity of an object by vibrating at a certain point(s), the stronger vibration the closer the object is. The operating time is 95 minutes at maximum. The data processing units are Arduino Pro-micro, Xbee module, myRIO device and PIC18F2550. Authors report that users require a training time to learn the device's operation. Scalability is not reported. This system is not oriented to the IoT.

The third one is a prototype that was proposed in India, consisting of a circuit made with Arduino Uno, a Bluetooth module and a single ultrasonic range sensor. The circuit is powered by a non-rechargeable battery Walimbe, A., et al., (2018). This circuit is accompanied by a mobile application developed for Android OS that vibrates and throws beeps depending on how far away an obstacle is encountered. 
However, we consider that this project was reported at an early stage, because there is no mention of adapting the circuit to a garment. In addition, it has a single HC-SR04/05 sensor, which was tested at Uitsiton, and demonstrated deficiencies regarding the performance established in the manufacturer's datasheet. The operating time is not reported in the article. Therefore, learning time and scalability are not reported. This system is not oriented to the IoT.

The fourth one is the Wearable MAPTIC Emilios Farrington-Arnas, (2019), it detects obstacles at the front of the user at chest height, through ultrasonic range sensors. It consists of a necklace and a series of feedback units that can be placed on clothing or around the wrist, such as a bracelet. The necklace detects obstacles and the bracelet vibrates left or right. MAPTIC wearable connects to a voice-controlled IOS App, so GPS can be used to guide the user. The MAPTIC website does not report operating time. It is important to mention that MAPTIC is a commercial product; therefore, there is no access to the hardware architecture or the architecture of the App. Learning time and scalability are not reported. Since it uses the smartphone current GPS capabilities and Google Maps API to provide the data for navigation, we consider that MAPTIC is oriented to the IoT.

Table 1 shows feature comparison of mentioned systems with ours. Uitsiton has some advantages over related works, such as: longer operating time, shorter learning time, better sensing coverture range and better scalability. Also, it is oriented to the IoT. Each of these advantages will be proved in this article.

\begin{tabular}{|c|c|c|c|c|c|}
\hline Feature & $\begin{array}{l}\text { Wearable } \\
\text { System }\end{array}$ & $\begin{array}{l}\text { Cinturón y } \\
\text { manitas }\end{array}$ & $\begin{array}{l}\text { Prototype } \\
\text { (India) }\end{array}$ & MAPIC & Uitsiton \\
\hline $\begin{array}{l}\text { Learning } \\
\text { time }\end{array}$ & $\begin{array}{l}2 \text { min } \\
\text { (Tested } \\
\text { in a } \\
\text { corrido } \\
\text { r) }\end{array}$ & $\begin{array}{l}\text { Require a } \\
\text { training } \\
\text { time }\end{array}$ & --- & --- & $\begin{array}{l}5 \mathrm{~min} \\
\text { (Tested } \\
\text { indoor } \\
\text { outdoor) }\end{array}$ \\
\hline Scalability & --- & --- & --- & --- & $\begin{array}{l}\text { It depends of } \\
\text { microcontrolle } \\
\text { r capability }\end{array}$ \\
\hline $\begin{array}{l}\text { Operating } \\
\text { time }\end{array}$ & $10 \mathrm{hrs}$. & $95 \mathrm{~min}$. & -- & --- & $20 \mathrm{hrs}$. \\
\hline $\begin{array}{l}\text { Sensing } \\
\text { coverture } \\
\text { range }\end{array}$ & $\begin{array}{l}\text { shoulde } \\
\text { r-height }\end{array}$ & 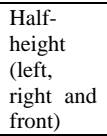 & --- & $\begin{array}{l}\text { Front of } \\
\text { the user } \\
\text { at chest } \\
\text { height }\end{array}$ & $\begin{array}{l}\text { Torso } \quad \text { (left, } \\
\text { right and } \\
\text { front) }\end{array}$ \\
\hline $\begin{array}{l}\text { Oriented to } \\
\text { the IoT }\end{array}$ & No & No & No & Yes & Yes \\
\hline
\end{tabular}

Table 1 Feature comparison of four systems with Uisiton Source: Prepared by the Authors

\section{Methodology \\ Observation and definition of the problem}

In general, the animals are guided by their senses to move in their environment. When the sense of the vision is affected the mobility is affected too. To improve the mobility, we need to substitute the vision of the impaired persons with some artificial sense, for example the audition combined with the tact can replace in some way the loosed natural vision. We need to resolve obstacle detection which is one of the main challenges. For this research project, the person's mobility in enclosed spaces and open spaces will be considered. The detection of holes or steps and this kind of open problems are not considered by us.

\section{Hypothesis}

It is possible to build an experimental wearable system with four aspects concerning usability: i) easy to learn, measured through learning time, ii) efficiency, measured through operating time, iii) sensing coverture range, and iv) scalability, measured by the possibility of increment the number of sensors. This experimental system will be oriented to the IoT.

\section{Experimentation}

- Study the movement in both close and open spaces of a person that cannot see.

- Based on the observation of at least 3 persons define where to set the 4 sensors and its disposition to sense at least 180 degrees in the walking direction.

- Design and build a first prototype to test the position and directions of the sensors, and dispose them correctly.

- Design and build the actuators component: Android OS App.

- Integrate the sensors component with the App.

- Test and document the preliminary results of the complete wearable.

- Fix possible errors or modify to improve the prototype. 


\section{Expected results}

- A wearable with 4 ultrasonic range sensors measuring the distance of objects at 180 degrees both to the front-horizontal and front-vertical of the center of the torso of the person that is wearing it.

- An Android OS App capable of: i) receiving signals from the electronic device from 4 sensors; (ii) process signals to decide about the existence of an obstacle in the movement direction; (iii) alert the visually impaired person through sounds and vibration patterns.

- To prove the hypothesis with the Uitsiton system in action.

\section{Development}

\section{Wearable design and implementation}

The wearable design and implementation consist of two components, embedded sensors system and Android OS App. Each one is presented below in two views: Logical and Physical.

\section{Embedded Sensors System}

Figure 1 shows the Logical View of Uitsiton's architecture, via a block diagram. The wearable consists of an array of 4 ultrasonic range sensors, a microcontroller, a Bluetooth module and a voltage regulator; the wearable requires a battery. Figure 2 shows the physical view concerning the connections between the electronic wearable components. The TRIGGER and ECHO pins of the four ultrasonic sensors were assigned to port $\mathrm{B}$ of the microcontroller. The data transmission and reception pins of the Bluetooth module were connected to the Rx and Tx ports, the microcontroller's EUSART module is located in the Bluetooth module. EUSART module supports some communication protocols, among them the RS-232. The voltage regulator protects the system from variations on levels of voltage.

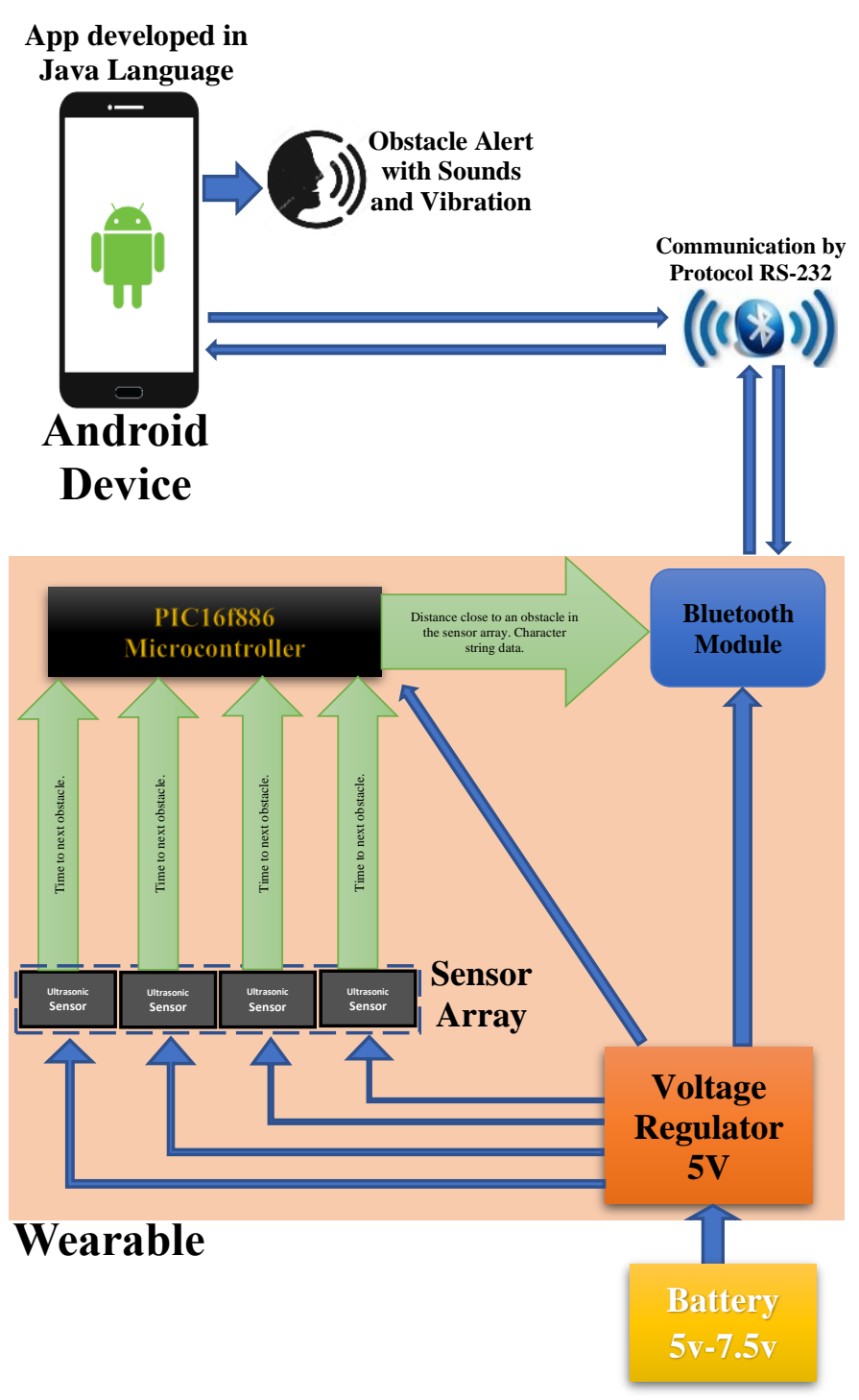

Figure 1 Uitsiton System Block Diagram: Logical View Source: Prepared by the Authors

The operation of ultrasonic range sensors consists of the cyclic emission of an ultrasonic wave, which spreads in the environment. When an object is found in its path, this wave is reflected and echoes to the receiver. The conditioning circuit computes the period between the emission of the ultrasonic waves and the reception of the reflected wave. The range of the wave is approximately 4.5 meters in ideal conditions. The 4 sensors disposition form a cross in the center of the person's torso, each of them has a different direction to their emission: left, right, up and down. The microcontroller records the time before wave emission and the return of the reflected wave. The microcontroller computes the 4 distances, in centimeters, using the difference between the emission and the return of the weaves timestamps, in microseconds, of each sensor. 
Subsequently, the microcontroller sends the distances to the Android App in bit frames via Bluetooth using the RS-232 protocol. Because of the simplicity of RS-232 is computational less expensive to operate on the PIC16F886 microcontroller, and it only requires two-way communication, which is achieved with three pins: Tx, Rx, and ground (GND). The lower the computational complexity in the microcontroller, the lower the energy consumption.

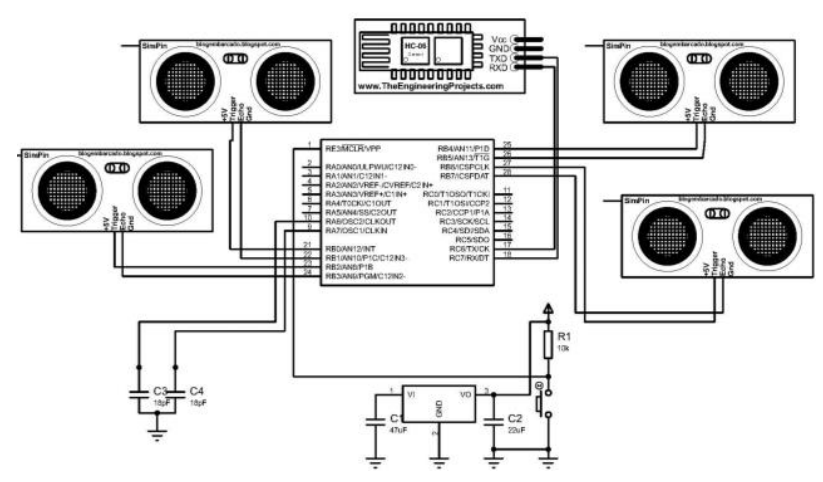

Figure 2 Wearable Electric Diagram: Physical View Source: Prepared by the Authors

The Figure 3 shows the implementation of the architecture of the Uitsiton's wearable. The board has a male header to make it an easyto-use test and experiments platform since it allows access to all pins of the microcontroller. The components are: two $22 \mathrm{pF}$ ceramic capacitors, male header's, resistor of $10 \mathrm{k} \Omega$, one push button and PIC6F886 microcontroller. The voltage regulator module is composed of: TTL LM2940T-05, 47 microfarads and 22 microfarad electrolytic capacitors. Before welding the components and regulator module to the board, all connections were tested for possible short circuits.

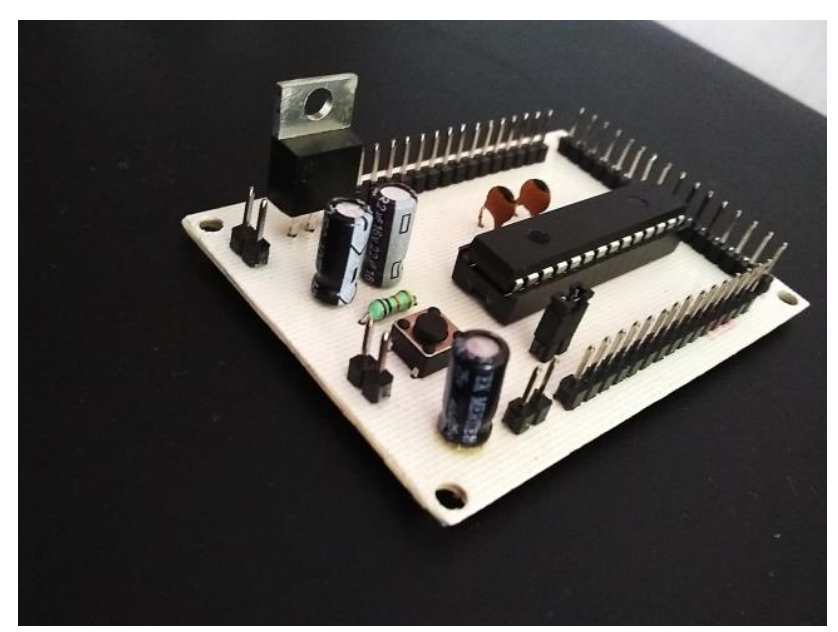

Figure 3 Implementation of the wearable architecture Source: Prepared by the Authors

\section{December 2020, Vol.6 No.18 7-18}

The goal of the algorithm implemented is to send the distances to the App at which potential obstacles are encountered. Initially the measured time of a first sensor is captured in the direction of the movement; immediately calculated the distance with equation 1 . Then the program waits 20 milliseconds and is passed and the second measured time of a second sensor; subsequently, the distance is calculated. And so on with the remaining sensors. Finally, the persisted distances are collected and sent to the App. The algorithm considers a limit of 1.20 meters to sense the obstacles. In addition, we experimentally found that to maintain a response time of 80 milliseconds, the range of the sensors had to be less than 1.50 meters. The response time for 4.5 meters obstacles is 150 milliseconds. Also, we found experimentally that in square rooms the reflected waves can make the device fail, because of the proximity of the walls. We decided to take as limit 1.20 meters to detect obstacles correctly in closed spaces with horizontal areas of 1.5 square meters.

$d[\mathrm{~cm}]=V\left[\frac{\mathrm{cm}}{\mathrm{s}}\right] t[s]$

Where $d$ is the distance, $V$ is the velocity of ultrasonic waves and $t$ is the time.

\section{App design and implementation}

The Logical View of Uitsiton's architecture in figure 1 shows the wearable communication with the App that will alert the user through sound patterns and vibration patterns. The App was designed under a two-layer architecture: view layer and business layer, figure 4 . The view layer is made up of three media: sound, vibration and graphical interface. The business layer performs data processing. It is important to mention that the App does not have a data layer because it does not require storing the bit frames it receives from the wearable. The App is composed of twelve classes, ten concrete and two interfaces, see the class diagram in Unified Modeling Language (UML) in figure 5. 


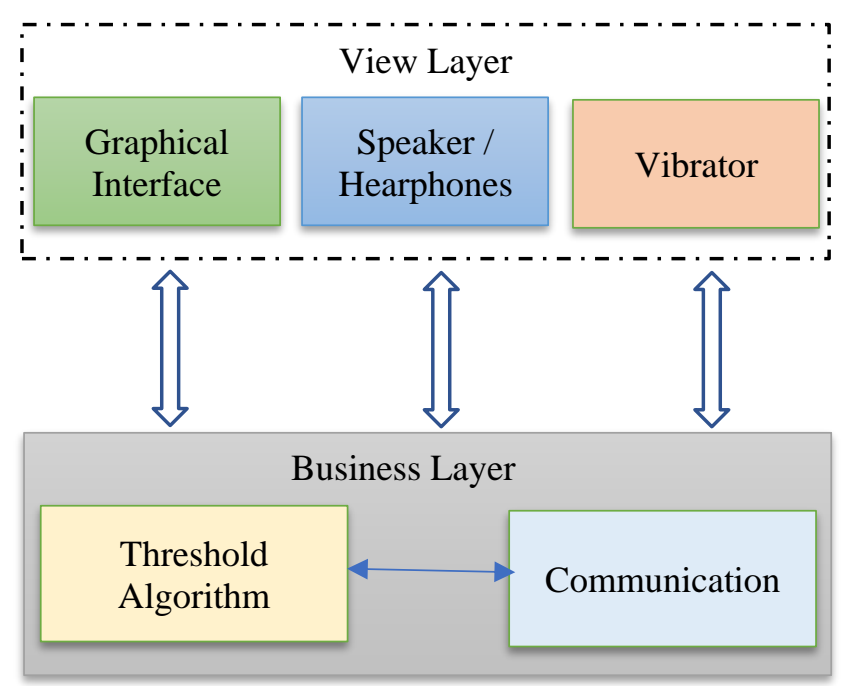

Figure 4 Two-Layer Architecture of the App: Logical View

Source: Prepared by the Authors

The Business Layer retrieves the bit frame sent by the wearable, through the MiHandler class. The received message is filtered to prevent incoherent bit frames. The filtered message is sent to the next logical component, SubProcesos class. The SubProcess class separates the message in tokens, which contains the distances of the sensors and assigns them respectively (parsing process). TheView Layer has the responsibility of alerting the user that some obstacle is in front of. This alert is made with sound patterns and vibration patterns, through the SonidoVibracionRunnable class. The View Layer also shows on the graphical interface the distances of the four sensors, through the TextViewRunnable class. The graphical interface has only sense for developers or testers, not for visual impaired users.

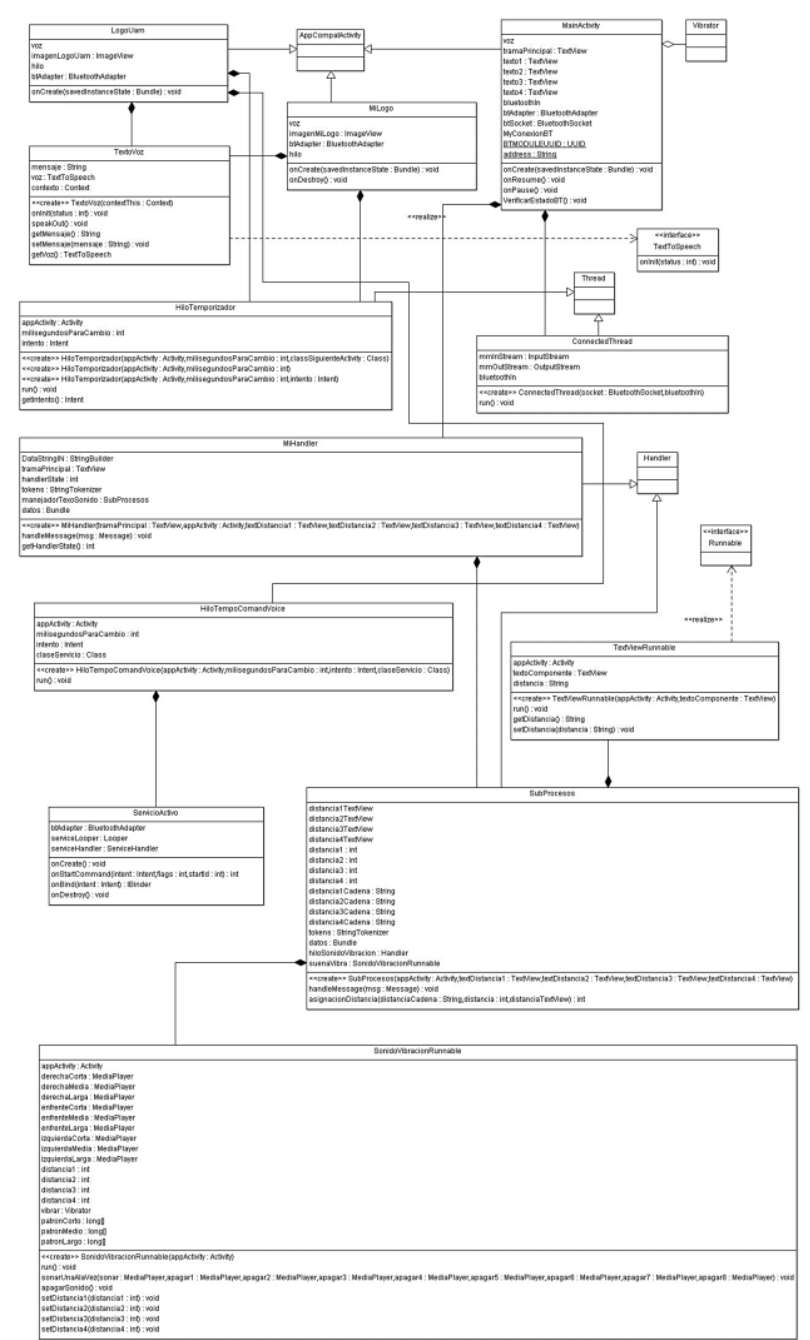

Figure 5 App Class Diagram in UML: Logical View Source: Prepared by the Authors

Figure 6 shows another view of the Uitsiton architecture. The Client-Server Model allows establishing the connection between the wearable and the App. The server lives in the Wearable and the client lives in the App. The connection is made via the Bluetooth API.

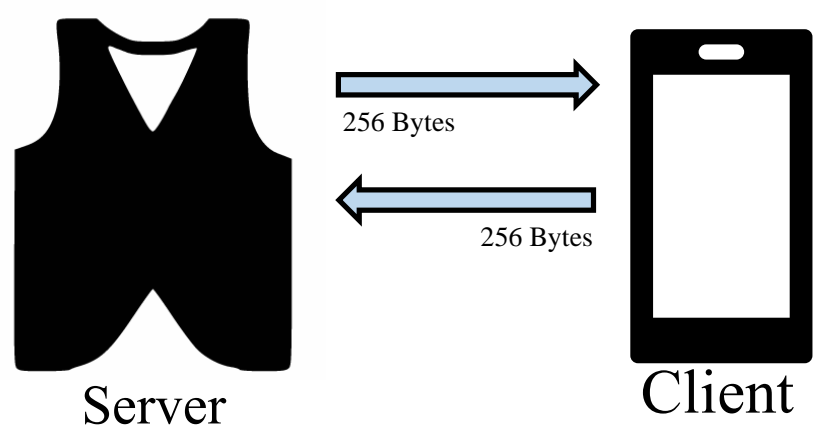

Figure 6 Client-Server Architecture: Physical View Source: Prepared by the Authors 
In the SonidoVibracionRunnable class, an algorithm dedicated to managing vibration and sound was implemented to alert the person. To apply the algorithm properly, sensor 1 (S1) should be attached to the right end of the chest; sensor 2 (S2) should be attached to the left end of the chest; sensor 3 (S3) should be placed on the upper right pectoral, near the clavicle; sensor 4 (S4) should be placed near the navel, between the left iliac fossa and the hypogastrium, as shown in figure 7.

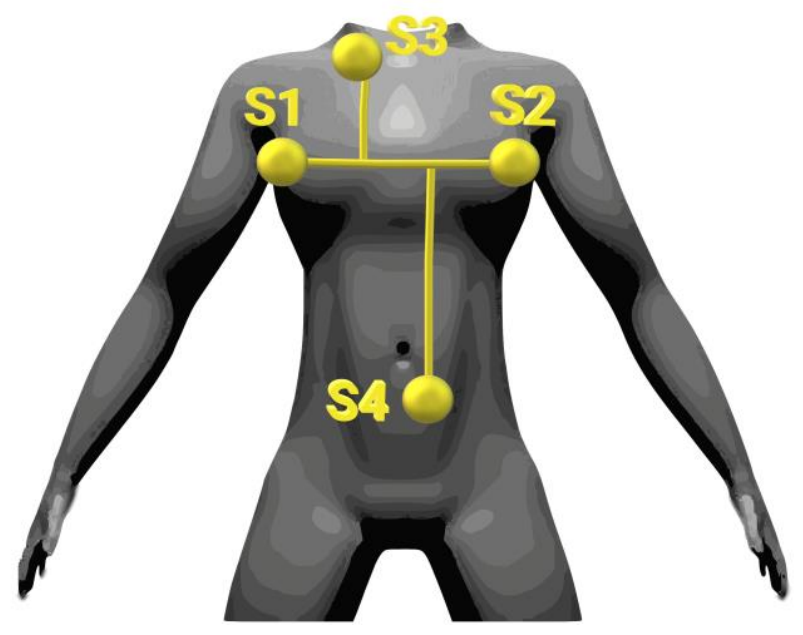

Figure 7 Disposition of sensors in the human corpus Source: Prepared by the Authors

The algorithm consists of comparing the 4 distances of the sensors and selecting the smallest. Once the smallest distance is selected, the algorithm assigns a specific vibration pattern and a specific sound pattern. For the right side a low tone was selected for the alert sound; while for the center a medium tone was selected, and for the left side was selected a high tone. The sound track was also accelerated to generate the effect of closeness to the obstacle; while it was slowed to generate the effect of remoteness of the obstacle. Therefore, the names of the sounds, shown in Table 2, have a qualifying adjective, namely: long, corresponding to far away; medium, corresponding to next, and short, corresponding to close. The vibration pattern will generate frequency intervals that also depend on the closeness or remoteness of the obstacle; therefore, the names of the vibration patterns, shown in Table 2, have a qualified adjective, namely: long, corresponding to far away; medium, corresponding to the next, and short, corresponding to close.

The vibration patterns generate on and off states with a constant intensity variation of the frequency depending on the distance of the obstacles. The Pattern_long vibrates with on and off intervals of $900 \mathrm{~ms}$.
The Pattern_medium vibrates with on and off intervals of $600 \mathrm{~ms}$; finally, the Pattern_short vibrates with on and off intervals of $400 \mathrm{~ms}$.

\begin{tabular}{|c|l|l|r|}
\hline \multicolumn{2}{|c|}{ Pattern Adjective } & \multicolumn{1}{c|}{$\begin{array}{c}\text { Range } \\
(\mathbf{c m})\end{array}$} \\
\hline Vibration & Right & Short & $(0,30)$ \\
& sound & Medium & {$[30,60)$} \\
& Left sound & Long & {$[60,120]$} \\
& In front & Mute /No & $(121, \infty)$ \\
& sound & vibration & \\
\hline
\end{tabular}

Table 2 Sound and Vibration Patterns Range with Adjectives

Source: Prepared by the Authors

The algorithm takes three thresholds, 30 , 60 and 120 centimeters, where 120 is the maximum distance of the model. These thresholds specify the following conditions to distance $x$ such as $x \in(0,30)$ or $x \in[30,60)$ or $x \in[60,120]$ or $x \in(121, \infty)$. The sensors 3 and 4 are encapsulated as "a single large sensor" that detects the central part of the torso because with this array we amplify the area of the sensing in the front, instead of an 30 degrees of sensing aperture like a cone shape, we create an united two cones like an number 8 shape. Therefore, the conditions to activate the sound and vibration are based on the combination of the distances found around the ranges, on each three sensors, i.e. 21 possibilities. Figure 8 shows 3 cases. In the case (a), the distance between $S 1$ and the obstacle, $\mathrm{x}<30$; therefore, the sound will be Right_short and the vibration will be Pattern_short. In the case (b), the distance between $S 34$ and the obstacle, $x<60$; therefore, the sound will be Infront_medium and the vibration will be Pattern_medium. In the case (c), the distance between $S 2$ and the obstacle, $60<\mathrm{x}<120$; therefore, the sound will be Left_long and the vibration will be Pattern_long.

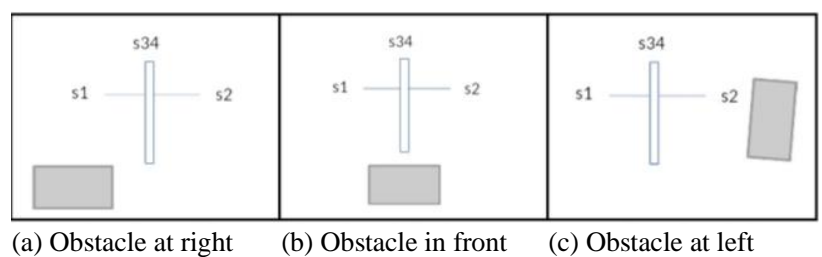

Figure 8 Three cases about the obstacles disposition Source: Prepared by the Authors 


\section{Uitsiton montage}

Figure 9 and 10 shows the front and rear views of the vest and shows the main circuit and batteries; Figure 11 displays the graphical interface of the App indicating the distance in centimeters provided by each of the four sensors; distance to which an obstacle lies at a certain time. Several types of frames or protectors were designed to protect both the main circuit and the ultrasonic sensors; the most suitable was chosen, and four 3D copies were subsequently printed.

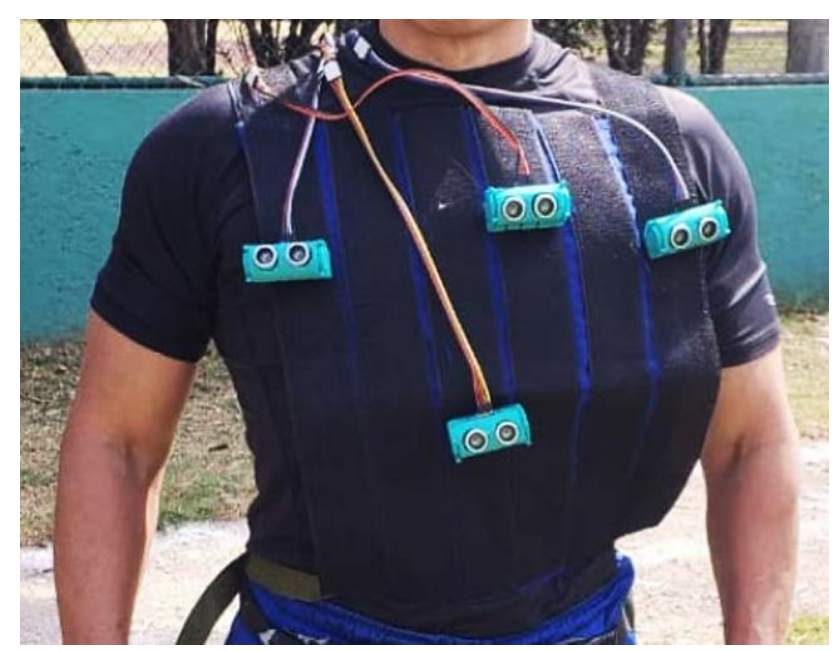

Figure 9 Wearable front view with sensors disposition Source: Prepared by the Authors

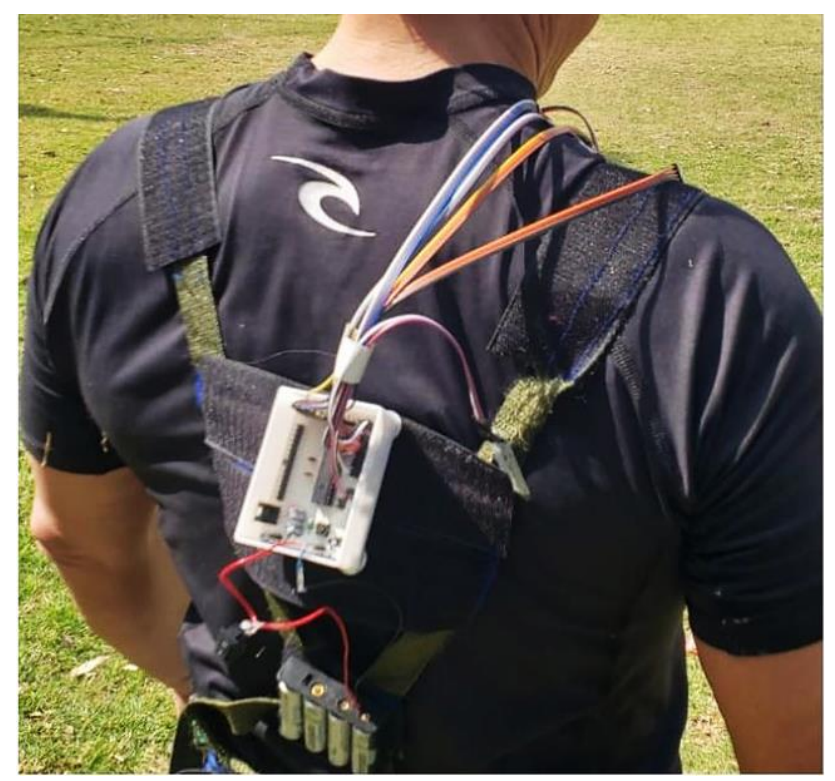

Figure 10 Wearable rear view showing main circuit and batteries

Source: Prepared by the Authors

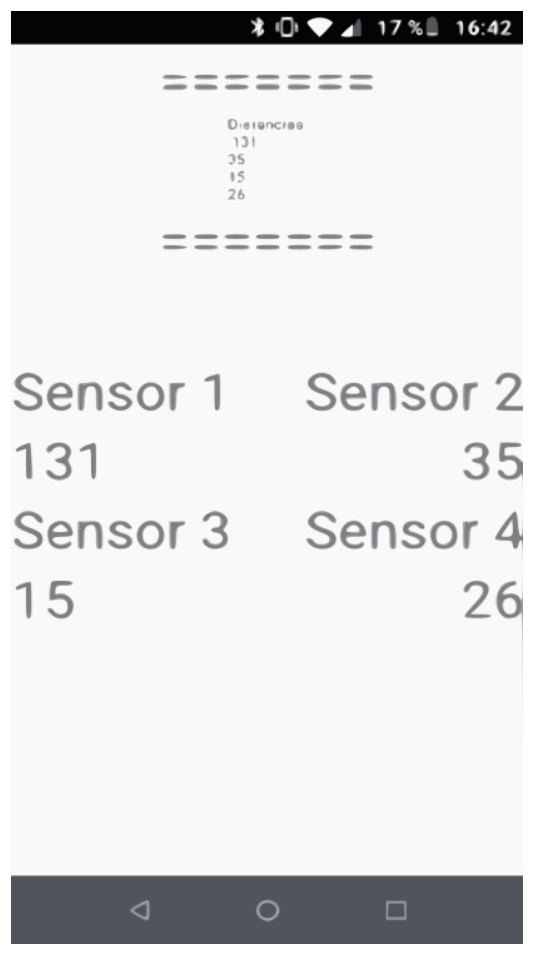

Figure 11 App's Graphical User Interface Source: Prepared by the Authors

\section{Test}

\section{Designing test}

\section{Cases}

Based on the cases shown in Figure 8 we have 4 cases, we can have obstacles at (a) right; (b) in front; at left (c); or a combination of (a), (b) and (c). Our test design laid on these 4 cases. We did various runs with 10 different persons wearing Uitsiton, each of them with the 4 cases.

\section{People}

We choose 10 participants, none of them have a visual impairment, but we consider that because these persons are not used to walking without seeing is more challenging for the Utsiton test than testing it with visually impaired persons. The age of the participants ranges from 20 to 55 years. They all use Apps on Android smartphones and were aware of Uitsiton at the time of the test.

\section{Terrain}

The person's movement in enclosed spaces and open spaces, and on smooth and uneven floors were considered. The enclosed and flat-floored places were room houses and offices. The open and uneven-floored places were universities, shopping plazas, and public parks.

BUENDÍA-MARTÍNEZ, Adrian \& GALLARDOLÓPEZ, Lizbeth. Uitsiton: wearable for mobility of the visually impaired. Journal of Scientific and Technical Applications. 2020 


\section{Obstacles}

In the house room were: chairs, tables, doors, walls, armchairs and furniture in general; obstacles in offices were other people, desks, pots with artificial plants, trash cans and different types of chairs. In the universities were: other people, trees, sidewalks, sign poles, sculptures, trash cans, tables and chairs. In the commercial plazas were: other people, trash cans, advertisement signs, plus shopping carts, and obstacles in public parks were trees, light poles, branches, tree roots, sidewalks, ground holes, deteriorated pavement and metal structures for different sport activities.

\section{Process}

Our process consists in preparation, mobility and interview of the participants. Preparation, the participant's eyes were covered with a piece of cloth; then the participants wore Uitsiton. The participant had to initialize the App with their eyes covered. Figure 12 shows a person who starts walking with the support of Uitsiton. Mobility, the participant was instructed to move from point $\mathrm{A}$ to point $\mathrm{B}$, following the four cases mentioned; this instruction was repeated several times, for an approximate time of 20 to 60 minutes, under constant supervision by the system manager. Interview, it was conducted at the end of the movement exercise, the participant provided his experience regarding the use of Uitsiton; participant also expressed his or her opinion on advantages, failures and improvements over the system.

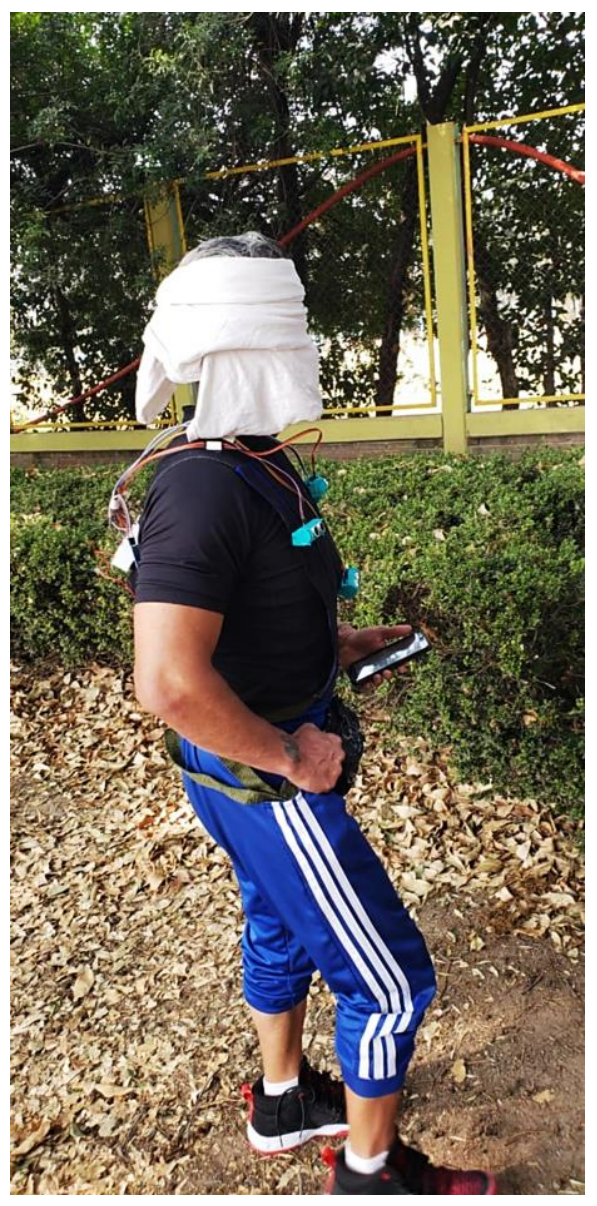

Figure 12 Uitsiton Tests: a person starts walking Source: Prepared by the authors

\section{Test Results}

\section{People}

When people were in places that had obstacles above their hips such as trees, advertisement signs, plant branches, sign poles, light poles, doors, tables and generally large furniture, their mobility was slow, but they felt confident with the system. People became acquainted, 5 minutes after they started walking, with the high, low and medium tones of the App, and their correspondence with the obstacle alert: Left_short, Left_medium, Left_long, Right_short, Right_medium, Right_long, In front_short, In front_medium, In front_long. The vibration of the smartphone reinforced the alert of the sound about the proximity of the obstacles. People said after 15 minutes they felt confident in Uitsiton, and lost the fear caused by the inability to see. Five people were even able to speed up the pace. 
People had difficulty placing the vest with their eyes covered. The velcro tape used to get stuck to other parts of the garment. The average time to place the vest for the first time was 6 minutes. People have different sizes, minimum 1.50 meters and maximum 1.80 meters; this was not a problem because the vest allows the sensors to be adjusted to properly direct them.

Occasionally, regardless of whether the App was installed on the participant's smartphone or if one was provided with the App installed, $37.5 \%$ of the time it was necessary to configure the device manually to activate Bluetooth. $62.5 \%$ were enough to activate the voice assistant, which is released by holding your finger pressed on the main menu button and giving the command to activate Bluetooth.

\section{System}

Uitsiton's response time, in obstacle detection, was almost instantaneous, about 80 milliseconds; often this response time was imperceptible to the participant, when he kept a moderate to slow walk. This response time was sometimes perceived by the participants who accelerated the pace.

The lateral ultrasonic sensors, in particular, proved to have a radiation pattern that exceeds 40 degrees each, so they detect obstacles with ease, and coupled with the central sensors, cover a range of 180 degrees vertically and horizontally with respect to the center of the torso, and with a range of 1.20 meters. However, sensor 4 had two drawbacks: (i) it detected the person's knees and calves as obstacles. This problem was solved, modifying the direction in which this sensor was pointing; ii) failed to accurately identify obstacles such as: pavement with thick cracks, tree stumps roots, sidewalks and holes; this problem was due to the different factors that alter the criterion of the algorithm to detect obstacles, such as the variety in the sizes of people, their way of walking, their posture, among others.

The tests were carried out for 5 days, two people per day. Uitsiton was used two consecutive days, for 8 hours each day. After the completion of the test, the system remained on to assess battery life, which was recharged on the third day to continue testing.

\section{Results and discussion}

Uitsiton is the result of this research, it is a vest type wearable that together with an Android smartphone App, alerts a visually impaired person to the presence of obstacles in their path.

\section{Wearable Vest}

The wearable detects obstacles in front of the center of the person's torso, this means that the maximum range of coverage is between the chin, shoulders and above the knees, with an angle of 180 degrees horizontally and vertically, with a range in obstacle detection, of 1.20 meters. However, Uitsiton could reach a maximum of 4.5 meters by slightly modifying the wearable code, and of course modifying the alert algorithm in the App. The implication of these changes would be an approximate delay of 150 milliseconds in response time. To maintain a response time of 80 milliseconds, the maximum range is 1.5 meters. The algorithm, however, considers a range of 1.20 meters in order to avoid confusion in enclosed spaces, where it might ring and vibrate all the time.

The operating time of the Uitsiton wearable is 20 continuous hours. Compared to related works, Cinturón y Manillas provides 95 minutes of operation, equivalent to $7.91 \%$ of Uitsiton's time; while Wearable System estimates 10 hours of operation, equivalent to $50 \%$ of Uitsiton's operating time. Uitsiton saves energy because the battery series required in the vest only powers the main circuit and ultrasonic sensors and the protocol implemented for the wireless communication is simple enough to be energy consumption optimum. Indeed, Uitsiton does not incorporate vibration motors into the vest because it would imply higher energy consumption, since each motor needs $70 \mathrm{~mA}$ to $110 \mathrm{~mA}$, in addition, a device that manages them may be required.

\section{App for Smartphone Android}

The Android smartphone App is capable of: i) receiving the bitframes from the wearable; (ii) process bitframes to compute the existence of an obstacle; (iii) alert the visually impaired person through sounds and vibration. One main feature is that the App can be launched by voice command, by simply pressing the main menu button on the smartphone for a few seconds. 
The App provides an experimentation interface, which verifies the correct initialization of the system; notifies, through the artificial voice of Android, the availability of Bluetooth, and shows distances to obstacles. The energy consumed by the App on the smartphone depends mainly on the Bluetooth connection and vibration motor, because the display is not necessary for the detection. During testing, we observed that the battery of the smartphones that ran the Uitsiton App with display on, decreased by $13 \%$ every hour on average.

About the algorithm, on one hand, the order of complexity of our algorithm is linear $O(n)$ because it executes only comparison and arithmetic operations. Based on the complexity of the algorithm we can say that the energy consumption is the minimum possible. Our algorithm does not require the display on and uses the bluetooth technology that has as an important characteristic its low power consumption. On the other hand, the algorithm was designed as a base for creating a platform for experimentation, thus this algorithm is extensible and highly configurable.

Based on the evidence we can say that Uitsiton is able to reveal obstacles such as signposts, advertisement signs, trees, walls, solid furniture, metal objects, among others; which is at less than or equal to 1.20 meters. However, based on the tests carried out, Uitsiton hardly detects amorphous objects with surfaces less than $2 \mathrm{~cm}^{2}$, also obstacles found at an altitude below the person's knee, such as stair steps. Uitsiton has difficulty accurately detecting obstacles such as: pavement with thick cracks, tree roots, sidewalks and holes; therefore, it should be used in conjunction with a cane or other mechanical instrument, when the visually impaired person is walking on uneven floors.

\section{Hypothesis Validation}

Based on preview sections we can say that it is possible to build an experimental wearable system with four aspects concerning usability: i) easy to learn, ii) efficiency, iii) sensing coverture range, and iv) scalability. This experimental system is oriented to the IoT.
About the ease of learning, testing shows that users took around 5 minutes to learn and 15 min to feel confident with Uitsiton; for people not used to not seeing, we consider these results as favorable to it. This result is comparable to the related systems, on Wearable System authors report 2 minutes for a user to learn the wearable in a closed place (corridor), while we report 5 minutes for a user to learn the Uitsiton system in both enclosed spaces and open spaces. About operation time, testing shows that Uitsiton has an operating time around 20 hours. This time exceeds $50 \%$ of the operating time of related systems. About sensing coverture range, Uitsiton detects obstacles that are at 180 degrees both to the front-horizontal and front-vertical; with the possibility of adjusting the sensors, in order to cover as much range as possible, depending on the size of the person who wears it. About scalability, Uitsiton's design makes it easy to add ultrasonic range sensors, many as the microcontroller allows. Since we design the programs in a modular way for both the microcontroller and the App, and the latter is loosely coupled with the wearable, our system is readily extensible. In addition, the vest makes it easy to modify the position of each sensor, and the sensor protectors make it easy to modify the direction in which each sensor points. Uitsiton is oriented to the IoT, thanks to use of smartphones, where it is possible to register data, about mobility as distance and time, in order to trace the displacements or monitor them remotely.

\section{Acknowledgements}

We thank Universidad Autónoma Metropolitana for the financial support for the publication of this article.

\section{Conclusions and future work}

It was possible to develop an experimental platform to study obstacle detection based on an IoT capable wearable system. This platform belongs to the ETA devices and uses the SONAR approach. We proved that the Uitsiton system has a remarkable usability to help people with visual impairment problems or persons that are losing their vision. Also, this prototype can be used for navigating dark or dangerous environments like mines, caves, fires, among others. 
We have proved that by using the SONAR in our system we can guarantee an acceptable level of confidence to the solid surface obstacle detection as well as a low production cost because the TTL technology makes it possible. Since Uitsiton is oriented to IoT, we could collect data that help us to understand the behavior of people with visual impairment in an urban environment and with this information improve their mobility at urban infrastructure. The design of Uitsiton allows us to offer, in future actualization of the platform, services that improve the quality of life for visually impaired persons like: geolocalization, guidelines to arrive at a place and alarm of panic, among others.

In the short term Uitsiton may be used in conjunction with a cane or other mechanical instrument when the person is walking down stairs or when walking on a surface with holes. In the medium term, the design of the vest could be improved; for example, a vest like that of personnel working in transport or in the police, to improve the placement time. A buzzer could be integrated into the vest, which would be bleeped to warn the person about the system initialization. As for the App, Bluetooth Low Energy mode could be implemented, which allows to maintain communication with the wearable, even if the Android device is at rest mode, in order to save more power on the smartphone battery. Also, in the App we could add the option to configure the criterion of the Obstacle Alert Algorithm; for example, that detection is done at exactly 1.50 meters instead of 1.20 meters. In the long term, Uitsiton could spread, integrating other sensors into the vest to improve confidence in the system. The App could use the GPS API to direct the person to their destination, informing them about street crossings, the presence of traffic lights, among others. In addition, another electronic instrument could be integrated to detect obstacles in the lower knees and calves; in this way, Uitsiton could detect the presence of stair steps, holes and cracks; all this to enhance the mobility of visually impaired people.

\section{References}

Brito, G. J. (2020). Sistema de visión egocéntrica de reconocimiento de objetos cotidianos para el apoyo a personas con movilidad reducida.
Cardin, S., Thalmann, D., \& Vexo, F. (2007). A wearable System for Mobility Improvement of Visually Impaired. Obtained from The Visual Computer:

https://link.springer.com/article/10.1007/s00371 $-006-0032-4$

Dakopoulos, D., \& Bourbakis, N. G. (2009). Wearable obstacle avoidance electronic travel aids for blind: a survey. IEEE Transactions on Systems, Man, and Cybernetics, Part C (Applications and Reviews), 40(1), 25-35.

Discant, A., Rogozan, A., Rusu, C., \& Bensrhair, A. (2007, May). Sensors for obstacle detectiona survey. In 2007 30th International Spring Seminar on Electronics Technology (ISSE) (pp. 100-105). IEEE.

Emilios Farrington-Arnas. (2019). MAPTIC. London. Obtained from: https://emilios.co.uk/portfolio-maptic.html

Gonzales-Lorence, A., Sánchez-Orduña, G. G., Ayala-Landeros, J. G., \& Román-Flores, S. E. (2020). Smart glasses for blind people. Retreived form Ecorfan Journal of Technological Prototypes.. 6-17: 32-38.

Grijalva, H. M. \& Mejía, H. M. (2016). Cinturón y Manillas Vibradores Ultrasónicos para No Videntes. Ibarra, Ecuador. Obtenido de Universidad Técnica del Norte: http://repositorio.utn.edu.ec/bitstream/1234567 89/5612/3/ARTICULO.pdf

Walimbe, A., Rao, S., Sureban, A., \& Shah, M. (2018). Survey on Obstacle Detection and its Notification through an Android App for Visually Impaired People. Obtained from Asian Journal For Convergence In Technology, 3(3): https://www.asianssr.org/index.php/ajct/article/ view/244

World Health Organization. (2014). 10 facts about blindness and visual impairment. Obtained from: https://www.who.int/features/factfiles/blindness /blindness_facts/en/index $2 . h t m l$ 